\section{Immunkonjugat nützt älteren AML-Patienten}

Jüngere Patienten mit akuter myeloischer Leukämie (AML) leben länger, wenn sie zusätzlich zur Induktionschemotherapie Gemtuzumab Ozogamicin (GO) erhalten. Profitieren auch ältere Patienten von dem Immunkonjugat?

W ährend bei jüngeren Patienten mit AML in den vergangenen Jahren das Überleben verbessert werden konnte, hat sich bei über 60 -Jährigen wenig getan, vor allem, weil sie eine Intensivierung der Therapie oft nicht mehr verkraften. Antikörper, die das zytotoxische Agens zielgerichtet in die Krebszellen bringen, könnten den Therapieeffekt verstärken, ohne die Toxizität zu vermehren. Dieses Therapieprinzip wird mit GO umgesetzt und hat sich bereits bei jüngeren Patienten bewährt.

Nun wurde es in einer weiteren großen randomisierten Studie an über 1.100 älteren AML-Patienten geprüft: Bisher unbehandelte Patienten mit AML oder Hochrisiko-MDS erhielten randomisiert entweder eine alleinige Induktion mit Daunorubicin und Ara-C oder Clofarabin $(\mathrm{n}=556)$ oder zusätzlich GO (3 mg/ $\mathrm{m}^{2}$ an Tag 1 des ersten Therapiezyklus; $\mathrm{n}=559$ ). Die Patienten waren 51-84 Jahre alt (median 67 Jahre). Primärer Endpunkt war das Gesamtüberleben.

Das Gesamtansprechen umfasste $69 \%$ Komplettremissionen (9\% mit unvollständiger hämatologischer Erholung) ohne signifikanten Unterschied zwischen dem GO- (70\%) und dem Kontrollarm (68\%). Die kumulative 3-Jahres-Inzidenz für ein Rezidiv war unter
GO signifikant um $22 \%$ reduziert (68 vs. $76 \%$; Hazard Ratio [HR] 0,78; $\mathrm{p}=0,007$ ). Möglicherweise profitieren Patienten mit schlechterem Performance-Status etwas stärker von GO. Während bei der 30- und 60-Tages-Mortalität kein signifikanter Unterschied zwischen den beiden Gruppen bestand, war das 3-JahresGesamtüberleben unter der zusätzlichen GO-Therapie signifikant höher (25 vs. $20 \%$; HR 0,87; p = 0,05).

Es kam unter der GO-Behandlung zu keinem größeren Anstieg der Toxizität.

Fazit: Die Zugabe von GO zur Induktionstherapie reduziert das Rezidivrisiko und verbessert auch bei älteren AML-Patienten das Überleben, ohne die Toxizität maßgeblich zu steigern. Judith Neumaier

Burnett KA et al. Addition of gemtuzumab ozogamicin to induction chemotherapy improves survival in older patients with acute myeloid leukemia. J Clin Oncol. 2012;30(32):3924-31.

\title{
Alternative zu Imatinib bei neu diagnostizierter CML geprüft
}

\section{Bei Patienten mit chronischer myeloischer Leukämie (CML) und Resistenz oder Unverträglichkeit gegenüber anderen Tyrosinkinasehemmern (TKI) war Bosutinib effektiv. Nun wurde der orale Src/Abl-TKI bei Patienten mit neu diagnostizierter CML in der chronischen Phase geprüft.}

A n der Phase-III-Studie BELA nahmen 502 Patienten mit neu diagnostizierter CML in der chronischen Phase teil. Sie erhielten randomisiert entweder Bosutinib (500 mg/d) oder Imatinib (400 $\mathrm{mg} / \mathrm{d})$. Beim primären Endpunkt vollständiges zytogenetisches Ansprechen nach zwölf Monaten unterschieden sich beide Gruppen nicht signifikant (70 vs. $68 \% ; p=0,601$ ), aber in den sekundären Endpunkten z. B. der medianen Zeit bis zum vollständigen zytogenetischen Ansprechen (Bosutinib 12,9 vs. Imatinib 24,6 Wochen; $\mathrm{p}<0,001$ ) und bis zu einem

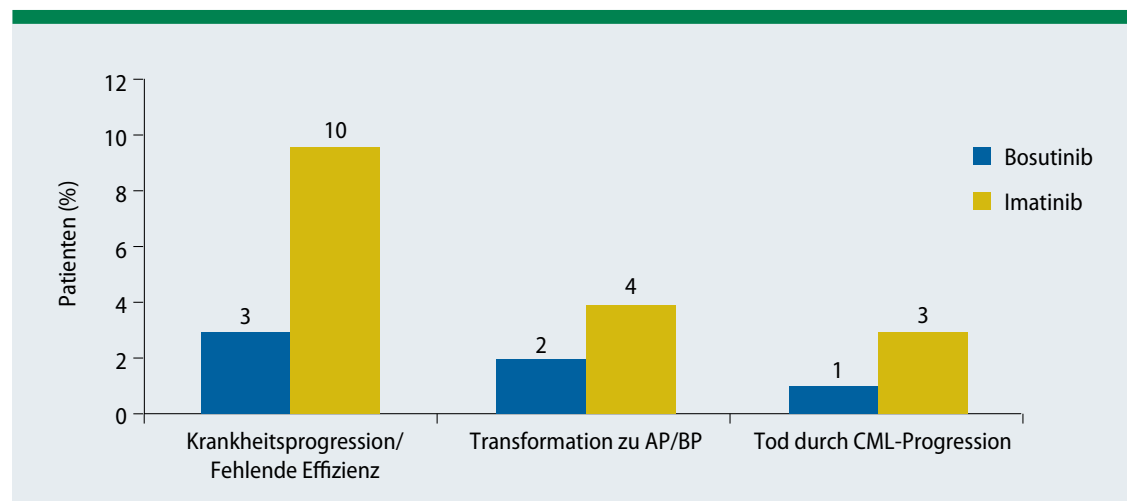

Abb.: Bei Patienten mit CML schnitt Bosutinib im Vergleich zu Imatinib in einigen sekundären Endpunkten besser ab. guten molekularen Ansprechen (37,1 vs 72,3 Wochen; $\mathrm{p}<0,001)$. Auch ein vollständiges bzw. gutes molekulares Ansprechen nach zwölf Monaten war unter Bosutinib signifikant häufiger als unter Imatinib (12 vs. $3 \%$; $<<0,001$ bzw. 41 vs. $27 \%$; $<0,001)$. Zum Übergang in die akzelerierte Phase bzw. Blastenkrise kam es bei vier Patienten (2\%) unter Bosutinib im Vergleich zu zehn (4\%) unter Imatinib.

Beide TKI hatten unterschiedliche Sicherheitsprofile: Während gastrointestinale und leberbezogene Ereignisse unter Bosutinib häufiger waren, traten unter Imatinib mehr Neutropenien, muskuloskeletale Symptome und Ödeme auf.

Fazit: In dieser Studie erreichte Bosutinib bei Patienten mit neu diagnostizierter CML in chronischer Phase den primären Endpunkt nicht. Allerdings schnitt es in verschiedenen sekundären Endpunkten signifikant besser ab als der bisherige Standard Imatinib.

Judith Neumaier

Cortes JE et al. Bosutinib versus imatinib in newly diagnosed chronic-phase chronic myeloid leukemia: Results from the BELA trial. J Clin Oncol. 2012;30(28):3486-92. 